\title{
THE PHYSICAL DISTORTION OF SCHMIDT PLATES
}

\author{
Paul Herget \\ Cincinnati Observatory
}

One characteristic of Schmidt telescopes is that the focal surface is spherical. This causes a flat photographic plate to be buckled and under strain while it is in the plate holder. In the early days of the space program, it was proposed to use Schmidt telescopes for the calibration of tracking stations because they were photographically fast. The writer opposed this proposal because they are not of high astrometric quality, for the reason indicated above.

Therefore an ordinary plate in the region of the Yale Zone Catalogue was borrowed from the 24-inch $(61 \mathrm{~cm}$.) Warner and Swasey Schmidt telescope of the Case Institute. Sixty-eight Yale stars were measured by hand on a Mann measuring engine, and the plate constants were determined as follows:

$$
\begin{aligned}
& x=a+b x+c y+d x y+e x_{2}^{2}+f x\left(x_{2}^{2}+y_{2}^{2}\right) \\
& x=a^{\prime}+b^{\prime} x+c^{\prime} y+d^{\prime} x y+e^{\prime} y^{\prime}+f^{\prime} y\left(x^{2}+y^{2}\right)
\end{aligned}
$$

A least squares solution was made from all the material. The residuals between the computed and the catalogue positions are as shown on the first slide. There is obviously a bad warp in the lower right hand region of the field. We then requested two more plates, each in a different plateholder, to be taken of the same region of the sky on the identically designed Curtis Schmidt telescope at the University of Michigan. The same procedure led to the results shown on the next two slides, using exactly the same catalogue positions of the comparison stars. You see that there is an entirely different pattern of the residuals.

In 1964 we had occasion to reduce forty-eight plates from the Palomar 48-inch $(122 \mathrm{~cm}$.) Schmidt, using the same procedure and about 150 comparison stars per plate, measured by C.J. van Houten. The large scale plot of the residuals showed no such obvious patterns of distortion, because the physical warping of the plates is relatively much less. The root-meansquare of the great circle residuals ranged from $0: 5$ to as high as $1: 3$ in a few cases. 